\title{
Microbial markers in the diagnosis of colorectal cancer: the promise, reality and challenge
}

\author{
Todd Z DeSantis ${ }^{1}$, Manasi S Shah², Julia L Cope ${ }^{3}$ \& Emily B Hollister ${ }^{*} 4,5$ \\ ${ }^{1}$ Informatics Department, Second Genome Inc., South San Francisco, CA 94080, USA \\ ${ }^{2}$ Algorithms \& Data Analysis, Microarray Business Unit, Thermo Fisher Scientific, Santa Clara, CA 95051, USA \\ ${ }^{3}$ Department of Scientific Operations, Diversigen, Inc., Houston, TX 77046, USA \\ ${ }^{4}$ Department of Pathology \& Immunology, Baylor College of Medicine, Houston, TX 77030, USA \\ ${ }^{5}$ Texas Children's Microbiome Center, Department of Pathology, Texas Children's Hospital, Houston, TX 77030, USA \\ * Author for correspondence: holliste@bcm.edu
}

\begin{abstract}
"Although the number of published studies suggesting that microbial features have diagnostic potential is encouraging, a general lack of consensus exists with respect to which features are most informative and/or from which microbial species they originate."
\end{abstract}

First draft submitted: 24 August 2017; Accepted for publication: 25 August 2017; Published online: 3 October 2017

Keywords: colorectal cancer • diagnostics • microbial markers

Colorectal cancer (CRC) is the second leading cause of cancer-associated mortality in the USA and the third most incident cancer globally [1]. Despite its frequent occurrence, 5-year CRC survival rates exceed $90 \%$ if the cancer is detected and treated at an early, localized stage [2]. Unfortunately, many individuals in high-risk groups are not screened $[1,3]$ since colonoscopy, the standard for CRC screening, is invasive and expensive. Noninvasive tests, like the guaiac-based fecal occult blood test, fecal immunochemical (FIT) test and DNA-based Cologuard test provide screening alternatives, but their utilization may be limited due to false-positive rates [1], need for repeat testing in the case of guaiac-based fecal occult blood test and FIT [1] and lack of sensitivity in detecting early-stage disease [1,4-5].

A variety of studies published in recent years have demonstrated that the gut microbiota associated with CRC differs from that of healthy individuals and, taken together, suggest that (fecal) microbial markers have the potential to supplement existing tests or provide alternatives for the noninvasive diagnosis of CRC [6-12]. The reported observations resulted from comparisons of CRC cases versus controls, using the abundances of sequencing reads from $16 S$ rRNA gene amplicons or shotgun metagenomics (see reference [8]).

\section{The reality}

Although the number of published studies suggesting that microbial features have diagnostic potential is encouraging, a general lack of consensus exists with respect to which features are most informative and/or from which microbial species they originate. For example, although Fusobacterium nucleatum is frequently detected in association with tumor tissue [11] and often reported as enriched in CRC tissues or having the potential to serve as fecal-based microbial marker for CRC [6-8], its identification as a diagnostic marker in CRC microbiome studies has not been universal [9-10,12-13]. This discordance may be a function of differences inherent among study populations, but interstudy variability with respect to procedures for fecal collection and storage, DNA extraction and amplification, sequencing and bioinformatics processing may also confound interpretation.

Lack of standardization with respect to study populations, sample collection and handling, as described above, is often the norm and is expected as technologies and the field mature. However, when data from disparate studies were processed using a standardized bioinformatics pipeline, greater concordance was observed among the studies and microbial markers were observed to significantly improve identification of CRC cases [13]. We have found that when CRC microbiome sequence data are processed in a uniform manner with a strain-resolved reference database,

Future : Medicine 
taxa other than F. nucleatum, including species within the genera Parvimonas, Porphyromonas and Parabacteroides appear to have greater potential to serve as diagnostic markers [13].

Most studies which have identified microbial markers with diagnostic potential do not limit their focus to 1-10 targets as do the FIT and Cologuard tests (i.e., hemoglobin and/or point mutations of $K$-ras and aberrant methylation of $N D R G 4$ and BMP3). Rather, they often highlight the fact that multiple taxa (i.e., 20 or more) serve as important contributors to classification accuracy $[6,8,13]$. These taxa typically comprise minority components of the microbiome, but taken together they appear to have good diagnostic potential.

The stochastic nature of capturing minority members of the gut microbiota through DNA sequencing [14] may help to explain why composite microbial biomarkers generally outperform single biomarkers in CRC classification. It also highlights the fact that no published study has yet to thoroughly and quantitatively assess all the microbial markers available in a given CRC fecal biospecimen. To date, all published studies have been limited to fewer than $10^{6}$ sequences per biospecimen, yet over $10^{12}$ sequences are likely extracted from a single fecal sample (estimated, $1 \mu \mathrm{g}$ of $500 \mathrm{bp}$ fragments $=1.8 \times 10^{12}$ sequences). In other words, less than 1 millionth of the available molecules are likely to have been surveyed. Biologists have accepted under sampling as a limitation of commonly available sequencing platforms, as have they accepted infrastructure-related limitations with respect to the computational resources required for the processing and storage of such volumes of data [15]. Under-sampling constraints have the potential to produce datasets in which minority taxa are only stochastically measured $[14,16]$ and present challenges with respect to reproducibility. Ideal methods to assess microbial biomarkers would be amenable to scale up to or beyond $10^{12}$ sequences, sensitive to both dominant and minority taxa, and able to generate reproducible, rather than stochastic, quantifications. Microbiome DNA-hybridization techniques offer potential solutions to each of these problems and have outperformed sequencing-based assays in identifying microbial markers relevant to bowel disease [17] and water contamination [18].

Microbial markers alone appear to provide similar classification accuracies as clinical features (i.e., $81-90 \%$ for microbial markers versus $81 \%$ for clinical features alone). However, when applied in combination, microbial markers and clinical characteristics (e.g., FIT or fecal occult blood test test results, age, sex, BMI) provide some of the best classification accuracies that have been reported to date (e.g., >90\%) [6,13]. Along similar lines, recent work evaluating the potential of the human gut virome (i.e., the viral community associated with the human GI tract) to distinguish CRC cases from healthy controls found that classification accuracy was highest when a combination of viral and bacterial markers was used [19]. Taken together, these results suggest that the 'microbial' CRC diagnostic may be a misnomer and that any microbial CRC diagnostic test is likely to rely on more than microbial features alone. A comprehensive, noninvasive CRC diagnostic test will likely rely on clinical or demographic information in combination with microbial features, including bacteria, viruses and perhaps, fungi.

\section{The challenge(s)}

Despite many of the limitations discussed here, microbial markers still show tremendous promise in the detection of CRC. However, a key limitation of microbial markers is that they do not necessarily perform as well in the detection of polyps, adenomas and other early-stage lesions as they do in the detection of more advanced disease [6-7,13]. If microbial marker-based diagnostics are to fulfill the promise of providing a noninvasive and relatively inexpensive means for CRC screening, they need to be able to accurately detect both early- and later-stage disease, as well as differentiate between stages. Data published to date suggest that there is still much room for improvement in this regard. In some cases, adenoma versus healthy and adenoma versus CRC classification rates, using bacterial markers, are comparable to differentiation of healthy versus CRC [19], but pooled analysis of fecal microbial profiles from adenoma cases suggests a reduced classification accuracy, sensitivity and specificity relative to comparisons of CRC versus healthy [13]. Importantly, different sets of microbial markers appear to be necessary for detection of the healthy versus adenoma transition, the adenoma versus CRC transition and the differentiation of CRC versus healthy [6-7,19]. Additional well-controlled studies are needed to support these efforts.

Several key and unaddressed challenges face the implementation of microbial marker-based tests for the diagnosis of CRC. Primary among these is the ability of such a test to detect CRC in individuals from high-risk populations who may already carry aberrant gut microbiota, such as those with irritable bowel disease (IBD), Type 2 diabetes and/or those who are overweight or obese. Although reported risk estimates vary, associations between IBD and CRC are well described, and individuals with longstanding IBD are generally acknowledged to be at greater risk for developing CRC [20]. Likewise, Type 2 diabetes and excess body weight are recognized risk factors for CRC [1], and $\mathrm{BMI}$ has proven to be useful in the development of microbially informed CRC classifier models $[7,13]$. The ability 
of a microbial marker-based CRC test to correctly distinguish between IBD or other disease-based aberrations in microbiota composition versus CRC-based ones will be critical for such a test to be adopted for routine screening of high-risk individuals.

Additionally, in order for a microbial marker-based test to be broadly applicable to the general public, the markers it includes must also be robust to the influences of common lifestyle factors which are known to influence gut microbiota composition. For example, it is recognized that the use of both prescription and over-the-counter medications, like antibiotics, proton-pump inhibitors, metformin, pain relievers and dietary supplements, may alter gut microbiota composition $[21,22]$. Gut microbiome studies often explicitly exclude participants with recent antibiotic use or chronic exposure to other medications to limit the confounding effects of these variables. As such, most or all of the microbial marker-based panels that have been developed and described to date still face the challenge of being validated under 'real-world' conditions. Validating potential microbial markers in the general population represents a critical step in confirming the accuracy and utility of microbial features in the diagnosis of CRC in the general public.

Although microbial markers show tremendous potential to augment or replace current noninvasive tests for the diagnosis of CRC, substantial hurdles remain to be cleared before we are likely to realize their full potential for routine CRC screening.

\section{Acknowledgements}

This editorial is dedicated to the inspirational life of B Atchinson.

\section{Financial \& competing interests disclosure}

TZ DeSantis is employed by Second Genome and holds stock and stock options in the company. MS Shah is an employee of Thermo Fisher Scientific and past employee of Second Genome. JL Cope is an employee of Diversigen, Inc. and holds stock and stock options in the company. EB Hollister has received honorarium payment from Roche Diagnostics, Inc., and TZ DeSantis, MS Shah and EB Hollister are named inventors on at least one pending patent application disclosing related subject matter. The authors have no other relevant affiliations or financial involvement with any organization or entity with a financial interest in or financial conflict with the subject matter or materials discussed in the manuscript apart from those disclosed.

No writing assistance was utilized in the production of this manuscript.

\section{Open access}

This work is licensed under the Attribution-NonCommercial-NoDerivatives 4.0 Unported License. To view a copy of this license, visit http://creativecommons.org/licenses/by-nc-nd/4.0/.

\section{References}

1. American Cancer Society. Colorectal Cancer Facts and Figures 2017-2019. (2017). www.cancer.org/content/dam/cancer$\mathrm{org} /$ research/cancer-facts-and-statistics/colorectal-cancer-facts-and-figures/colorectal-cancer-facts-and-figures-2017-2019.pdf

2. Howlander N, Noone AM, Krapcho M et al. SEER Cancer Statistics Review, 1975-2013. (2016). https://seer.cancer.gov/csr/1975_2013/

3. Centers for Disease Control and Prevention (CDC). Vital signs: colorectal cancer screening, incidence, and mortality - United States, 2002-2010. MMWR 60(26), 884-889 (2011).

4. Hundt S, Haug U, Brenner H. Comparative evaluation of immunochemical fecal occult blood tests for colorectal adenoma detection. Ann. Intern. Med. 150(3), 162-169 (2009).

5. Imperiale TF, Ransohoff DF, Itzkowitz SH et al. Multitarget stool DNA testing for colorectal-cancer screening. N. Engl. J. Med. 370(14), 1287-1297 (2014).

6. Baxter NT, Ruffin MTT, Rogers MA, Schloss PD. Microbiota-based model improves the sensitivity of fecal immunochemical test for detecting colonic lesions. Genome Med. 8(1), 37 (2016).

7. Zackular JP, Rogers MaM, Ruffin MT, Schloss PD. The human gut microbiome as a screening tool for colorectal cancer. Cancer Prev. Res. 7(11), 1112-1121 (2014).

8. Zeller G, Tap J, Voigt AY et al. Potential of fecal microbiota for early-stage detection of colorectal cancer. Mol. Syst. Biol. doi:10.15252/msb.20145645 (2014) (Epub ahead of print).

9. Wu N, Yang X, Zhang R et al. Dysbiosis signature of fecal microbiota in colorectal cancer patients. Microb. Ecol. 66(2), 462-470 (2013).

10. Weir TL, Manter DK, Sheflin AM, Barnett BA, Heuberger AL, Ryan EP. Stool microbiome and metabolome differences between colorectal cancer patients and healthy adults. PLoS ONE 8(8), e70803 (2013). 
11. Kostic AD, Gevers D, Pedamallu CS et al. Genomic analysis identifies association of Fusobacterium with colorectal carcinoma. Genome Res. 22(2), 292-298 (2012).

12. Wang T, Cai G, Qiu Y et al. Structural segregation of gut microbiota between colorectal cancer patients and healthy volunteers. ISME J. 6(2), 320-329 (2012).

13. Shah MS, Desantis TZ, Weinmaier $\mathrm{T}$ et al. Leveraging sequence-based faecal microbial community survey data to identify a composite biomarker for colorectal cancer. Gut doi: 10.1136/gutjnl-2016-313189 (2017) (Epub ahead of print).

14. Leray M, Knowlton N. Random sampling causes the low reproducibility of rare eukaryotic OTUs in Illumina COI metabarcoding. PeerJ 5, e3006 (2017).

15. Probst AJ, Lum PY, John B et al. Microarray of $16 \mathrm{~S}$ rRNA gene probes for quantifying population differences across microbiome samples. In: Microarrays: Current Technology, Innovations and Applications, He Z (Ed.). Caister Academic Press, Norwich, England, UK (2014).

16. Zhou J, Jiang Y-H, Deng Y et al. Random sampling process leads to overestimation of $\beta$-diversity of microbial communities. $m$ Bio $4(3)$, e00324-13 (2013).

17. Miezeiewski M, Schnaufer T, Muravsky M et al. An in vitro culture model to study the dynamics of colonic microbiota in Syrian golden hamsters and their susceptibility to infection with Clostridium difficile. ISME J. 9(2), 321-332 (2015).

18. Cao Y, van de Werfhorst LC, Dubinsky EA et al. Evaluation of molecular community analysis methods for discerning fecal sources and human waste. Water Res. 47(18), 6862-6872 (2013).

19. Hannigan GD, Duhaime MB, Ruffin MT, Koumpouras CC, Schloss PD. Viral and bacterial communities of colorectal cancer. bioRxiv doi:10.1101/152868 (2017) (Epub ahead of print).

20. Kim ER, Chang DK. Colorectal cancer in inflammatory bowel disease: the risk, pathogenesis, prevention and diagnosis. World J. Gastroenterol. 20(29), 9872-9881 (2014).

21. Zhernakova A, Kurilshikov A, Bonder MJ et al. Population-based metagenomics analysis reveals markers for gut microbiome composition and diversity. Science 352(6285), 565 (2016).

22. Rogers MA, Aronoff DM. The influence of non-steroidal anti-inflammatory drugs on the gut microbiome. Clin. Microbiol. Infect. 22(2), 178 e171-179 (2016). 